Received: 08.10.2021

Revised: 08.12.2021

Accepted: 24.12 .2021

DOI: $10.17804 / 2410-9908.2021 .6 .032-036$

\title{
IMPLEMENTATION OF DIGITAL METHODS TO ANALYZE EDDY-CURRENT SIGNALS BASED ON THE E14-440 MODULE
}

\author{
D. G. Ksenofontov ${ }^{1, a)} *$ and V. N. Kostin ${ }^{1,2}$ \\ ${ }^{1}$ M.N. Miheev Institute of Metal Physics, Ural Branch of the Russian Academy of Sciences, \\ 18 S. Kovalevskoy St., Ekaterinburg, 620208, Russia \\ ${ }^{2}$ B.N. Yeltsin Ural Federal University, Ekaterinburg, \\ 19 Mira St., 620002, Russia \\ a) (iD http://orcid.org/0000-0002-4526-0477 ksenofontov@imp.uran.ru; \\ b) iD http://orcid.org/0000-0003-4651-5002 kostin@ imp.uran.ru \\ *Corresponding author: E-mail: ksenofontov@imp.uran.ru \\ Address for correspondence: 18, S. ul. Kovalevskoy, Ekaterinburg, 620108, Ekaterinburg, Russian Federation \\ Tel.: +7 (343) 3783850
}

Nondestructive testing methods utilize various sensors, and different signal types require different processing methods. Digital implementation of signal processing methods can expand the variety of methods implemented by one system. An eddy-current test system based on the E14-440 module has been developed. Quadrature amplitude demodulation and fast Fourier transformation are implemented to analyze the signal. The amplitude, phase, and complex parts of the signal are calculated. It is shown that both methods are applicable and allow elimination of some analog circuits. However, digital signal processing significantly depends on conversion rates and synchronization between generation and pickup of the signal.

Keywords: Eddy-current, digital signal processing, amplitude, phase.

\section{Introduction}

Multipurpose hardware-software systems for nondestructive testing and evaluation have great potential [1]. Each nondestructive method employs a great number of different sensors that require corresponding signal processing circuits. Such circuits can represent amplification, signal processing, filtration, etc. However, some signal processing methods can be implemented digitally. These methods are Fourier transformation, signal modulation, filtration, and others. In this way, one device with unified hardware and different software is able to implement a wide range of nondestructive testing methods. Yet, such devices require further investigation.

\section{Methods and materials}

One of the key signal processing methods in eddy-current testing is quadrature amplitude modulation [2], which allows the evaluation of the amplitude and phase of the signal. A typical circuit is shown in Fig. 1. Such circuits usually process sinusoidal waves with a single frequency. This scheme provides only four signal characteristics: amplitude, phase, as well as real and imaginary parts of the signal. Moreover, it is complicated to analyze the initial signal from the coil after demodulation and filtration. Thus, pulsed eddy-current techniques often employ signals collected in the air or on the surface of a non-defective specimen as the reference signal [3].

To perform tests, an E14-440 high-speed external input/output module with a USB interface was used. The most significant components are an analog-to-digital converter (ADC), a digital- 
to-analog converter, a programmable amplifier, and a USB interface. The main characteristics of the module are described in Table 1.

Measurements were carried out under the following settings:

- $\mathrm{ADC}$ rate was set to a maximum of $400 \mathrm{kHz}$ to expand the range of signal frequencies;

- DAC rate was set to $100 \mathrm{kHz}$. Thus there are four ADC counts per DAC count;

- The refresh rate of most devices ranged between 60 and $120 \mathrm{~Hz}$. Thus the sampling rate should correspond to these values. The buffer size must be a power of 2 . For the ADC buffer size of 4096 samples, the data refresh rate was $96.75 \mathrm{~Hz}$. DAC buffer size was smaller by a factor of 4 , respectively.

The aforementioned settings allow analyzing signals in frequencies between $97.67 \mathrm{~Hz}$ and $12.5 \mathrm{kHz}$. The frequency range is mostly limited by the DAC rate and the DAC buffer size.

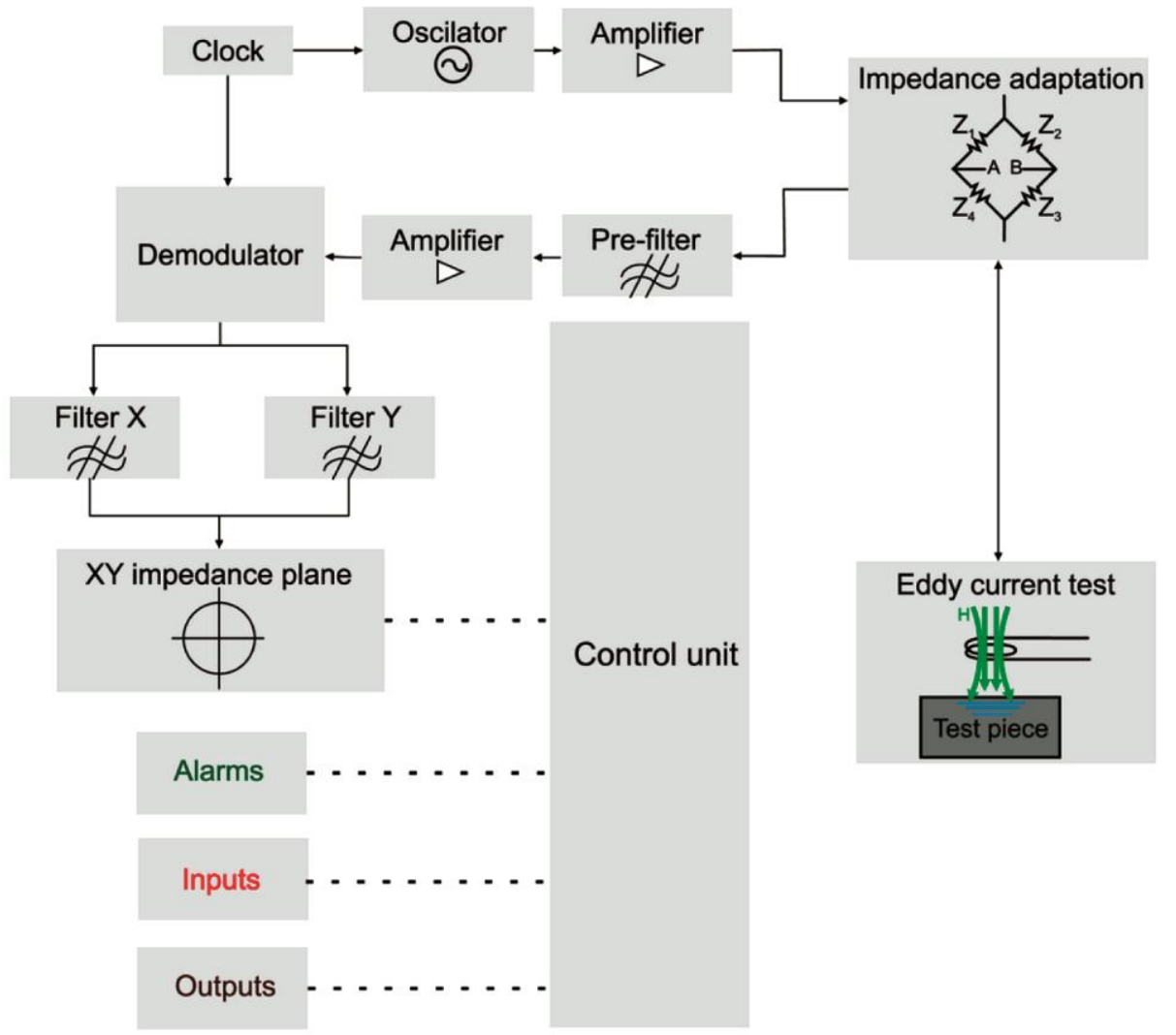

Fig. 1. Block diagram of an analog eddy-current system [2]

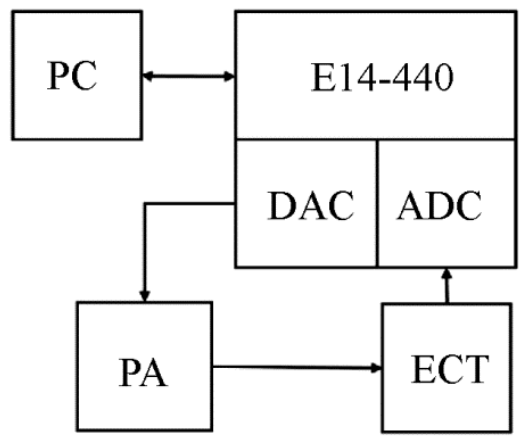

Fig. 2. Block diagram of the test eddy-current system. PC - personal computer,

$\mathrm{PA}$ - power amplifier, ECT - eddy-current transducer 
An encircling coil probe was used for testing. The driving coil has 700 turns of $0.25 \mathrm{~mm}$ wire and the pickup coil has 1000 turns of 0.10 wire. The inner diameter of the coils is $11 \mathrm{~mm}$, the length is $80 \mathrm{~mm}$. A standard amplifier scheme based on TDA2006 is used to provide current to the driving coil. The pickup coil is directly connected to the analog input of the module as far as it has an internal voltage amplifier. The voltage of the pickup coil is widely used as a signal for various applications [4-6]. The block diagram of the test eddy-current system is shown in Fig. 2.

Table 1. Specifications of the E24-440 module

\begin{tabular}{|c|c|c|}
\hline Parameter & Typical Value & Units \\
\hline Number of channels (ADC+DAC) & $16+2$ & - \\
\hline ADC resolution & 14 & bit \\
\hline Input ranges & $\pm 10 \mathrm{~V} ; \pm 2.5 \mathrm{~V} ; \pm 0.625 \mathrm{~V} ; \pm 0.156$ & $\mathrm{~V}$ \\
\hline Maximum ADC rate & 400 & $\mathrm{kHz}$ \\
\hline DAC resolution & 12 & bit \\
\hline Output range & \pm 5 & $\mathrm{~V}$ \\
\hline Maximum DAC rate & 125 & $\mathrm{kHz}$ \\
\hline
\end{tabular}
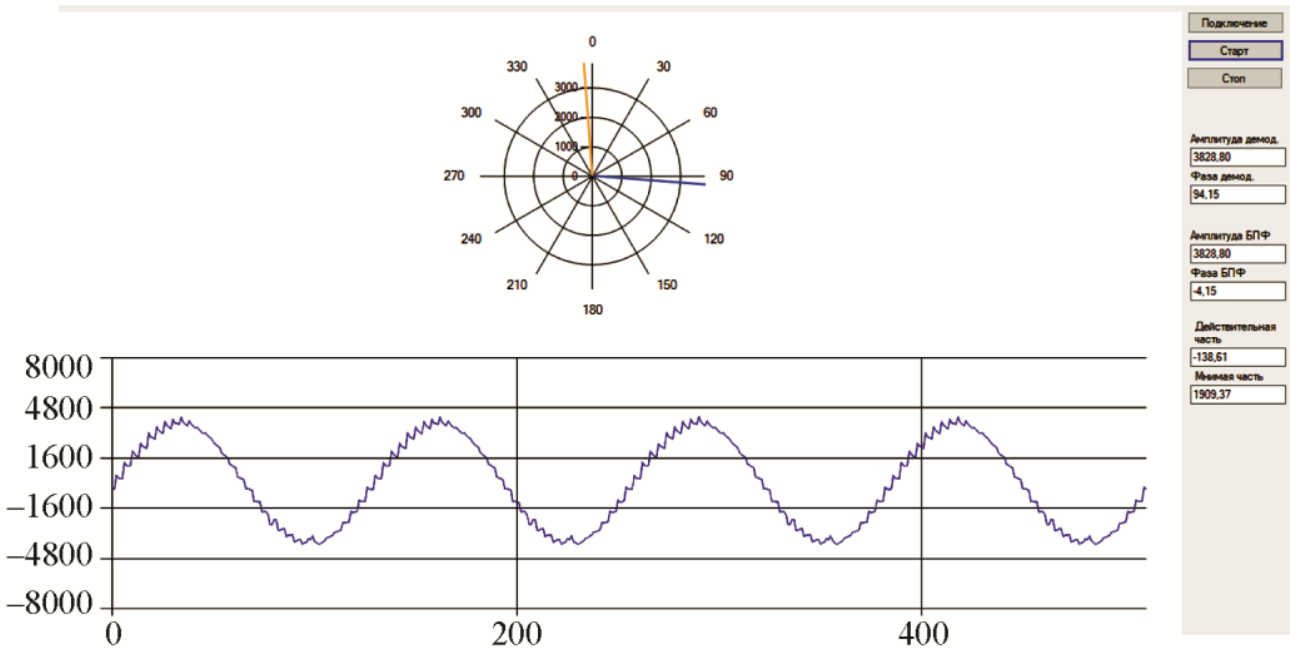

Fig. 3. The graphical user interface of the developed software

Corresponding software was developed to analyze the signal. Quadrature amplitude demodulation (QAD) and fast Fourier transformation (FFT) [7] algorithms were used. The software user interface is represented in Fig. 3. At the upper left part of the interface, the signal is displayed in polar coordinates. At the lower left part of the interface, a signal is displayed in amplitude and time coordinates. The right part of the interface displays amplitude and frequency calculated with QAD and FFT, real and imaginary parts calculated with QAD.

Table 2. Specimen properties

\begin{tabular}{|c|c|c|c|c|}
\hline Number & Material & Diameter, $\mathrm{mm}$ & $\begin{array}{c}\text { Resistivity, } \\
10^{-8} \Omega \cdot \mathrm{m}\end{array}$ & $\begin{array}{c}\text { Conductivity, } \\
\mathrm{MS} / \mathrm{m}\end{array}$ \\
\hline 1 & Brass & 10.02 & 6.49 & 15.42 \\
\hline 2 & Aluminum & 9.57 & 5.67 & 17.63 \\
\hline 3 & Aluminum & 9.87 & 5.76 & 17.36 \\
\hline 4 & Steel & 10.05 & 11.47 & 8.72 \\
\hline 5 & Steel & 9.97 & 14.20 & 7.04 \\
\hline
\end{tabular}


Five cylindrical specimens of different materials were used. Electrical conductivity was measured with the four-point method. The properties of the specimens are presented in Table 2 . The electrical conductivity of the aluminum specimens is lower than expected.

\section{Results}

The absolute values of amplitude, phase, and real and imaginary parts of the signal with different specimens have been measured for different signal frequencies. The E14-440 module has a random delay between the start of the ADC and the DAC. Therefore, the starting values of the phase, real and imaginary parts of the signal are also random. While the ADC and DAC are started, one can measure the change between values with and without specimens. Both the starting value and the value change should be stable. Random delay leads to random relative change in real and imaginary parts for every start of the system. Thus the absolute amplitude value, the absolute amplitude change, and the absolute phase change can be used as meaningful parameters of eddy-current testing for the present system.

The amplitude values calculated with QAD and FFT are equal. The phase values calculated with these methods differ by $90^{\circ}$. On the one hand, QAD allows calculating complex parts of the signal. On the other hand, FFT provides the signal specter. In this way, both methods are valuable, and they should be implemented jointly.

The absolute amplitude changes and the absolute phase changes calculated with QAD for the range of signal frequencies for different specimens are shown in Fig. 4.

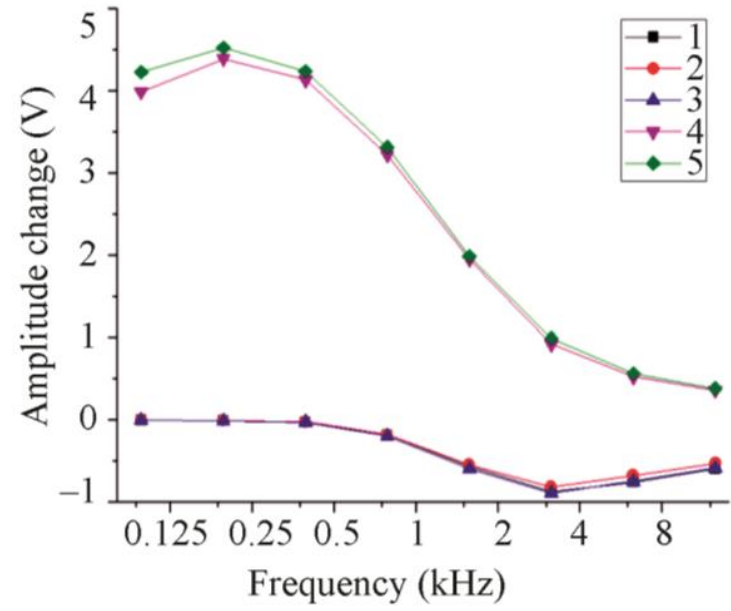

a

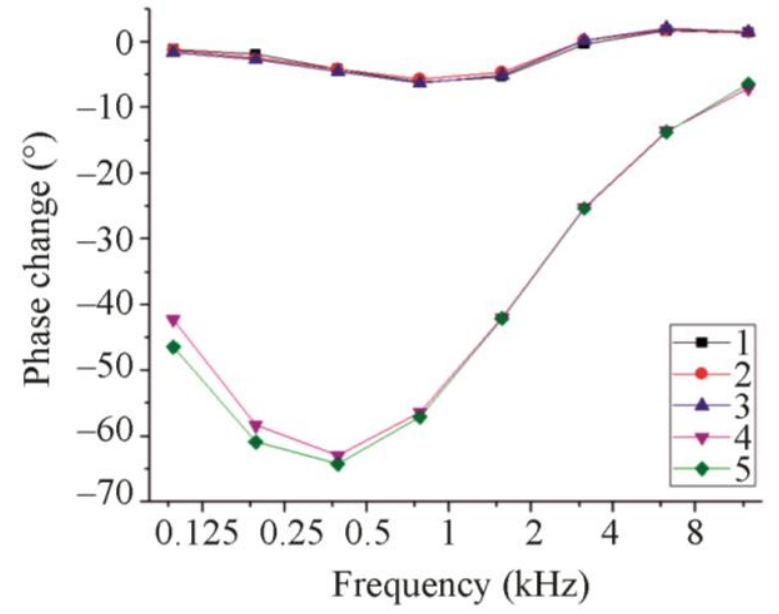

b

Fig. 4. Absolute amplitude change (a) and absolute phase change (b) for the specimens in the frequency range from $97.67 \mathrm{~Hz}$ to $12.5 \mathrm{kHz}$

The observed changes differ for ferromagnetic $(4,5)$ and non-ferromagnetic (1-3) specimens in the amplitude value, the phase value, the amplitude sign, the phase sign, and peak frequencies. The ferromagnetic specimens show significant amplitude and phase change at low frequencies. The most suitable frequency to distinguish ferromagnetic objects is $97.67 \mathrm{~Hz}$. Non-ferromagnetic specimens show less significant change in parameters with higher peak frequency. It is also difficult to distinguish non-ferromagnetic objects by amplitude or phase. A more significant difference among the specimens might be expected at even higher frequencies of excitation signal, which is above maximum of the developed system. Therefore, normalized or complex parameters should be used. 


\section{Discussion and conclusion}

The test system based on an E14-440 module has been developed. Quadrature amplitude demodulation and fast Fourier transformation have been employed in digital signal processing at frequencies of $97.67 \mathrm{~Hz}$ to $12.5 \mathrm{kHz}$. Specimens made of aluminum, brass, and steel have been tested. The developed system provides observation of significant amplitude and phase changes of the signal for various materials, which can be used for material characterization.

The implemented digital methods of signal processing are believed to eliminate the need for analog demodulators and low pass filters. They expand the range of analyzed signals. However, the methods significantly depend on DAC and ADC rates and processing capabilities of processors and microcontrollers used.

\section{Acknowledgments}

This work was carried out under the state assignment from the RF Ministry of Education and Science, themes Diagnostics, project no. AAAA-A18-118020690196-3, and Expertise, project no. AAAA-A19-119062590007-2.

\section{References}

1. Kostin V.N., Smorodinskii Ya.G. Multipurpose software-hardware systems for active electromagnetic testing as a trend. Russian Journal of Nondestructive Testing, 2017, vol. 53, No. 7 , pp. 493-504. DOI: 10.1134/S1061830917070075.

2. García-Martín J., Gómez-Gil J., Vázquez-Sánchez E. Non-Destructive Techniques Based on Eddy Current Testing. Sensors, 2011, vol. 11, No. 3, pp. 2525-2565. DOI: 10.3390/s110302525.

3. Zemin C., Mengbao F., Binghua C., Bin H., Jingwei S. Characterization of image sequences of a defect using pulsed eddy current signals. Journal of Magnetism and Magnetic Materials, 2021, vol. 534, pp. 168007. DOI: 10.1016/j.jmmm.2021.168007.

4. Wang Y., Fan M., Cao B., Ye B., Wen D. Measurement of coating thickness using lift-off point of intersection features from pulsed eddy current signals. NDT \& E International, 2020, vol. 116, pp. 102333. DOI: 10.1016/j.ndteint.2020.102333.

5. Wang T., Wu D., Chen W., Yang J. Detection of delamination defects inside carbon fiber reinforced plastic laminates by measuring eddy-current loss. Composite Structures, 2021, vol. 268, pp. 114012. DOI: 10.1016/j.compstruct.2021.114012.

6. Mizukami K., Hioki S., Moriyama K., Ogi K., Miyaji W., Kimura K. Eddy-current array-probe technique for imaging near-surface and deep-lying delaminations in multidirectional carbon fiber composites. Composite Structures, 2021, vol. 276, pp. 114537. DOI: 10.1016/j.compstruct.2021.114537.

7. Tretter S.A. Communication System Design Using DSP Algorithms, College Park, MD, Springer US, 2008, $352 \mathrm{p}$. 